\title{
Dynamic Stability Critical State of Pin-Ended Arches under Sudden Central Concentrated Load
}

\author{
Kai QIN*, Jingyuan LI**, Mengsha LIU***, Jinsan JU**** \\ *College of Civil Engineering and Architecture, Zhejiang University, Yuhangtanglu No. 866, Xihu District, Hangzhou, \\ Zhejiang 310058, China \& BIAD Co., Ltd, No 62. Nan Li Shi Lu Xicheng District, Beijing 100045, China, \\ E-mail:1454421@qq.com \\ **Department of Civil Engineering, Tsinghua University, Qinghuayuan No.1, Haidian District, Beijing 100084, China, \\ E-mail: lijingyuan0909@163.com \\ ***College of Water Resources and Civil Engineering, China Agricultural University, Qinghuadonglu No.17, Haidian \\ District, Beijing 100083, China, E-mail: liumengsha@cau.edu.cn \\ ****College of Water Resources and Civil Engineering, China Agricultural University, Qinghuadonglu No.17, Haidian \\ District, Beijing 100083, China, E-mail: jujinsan@cau.edu.cn (Corresponding Author)
}

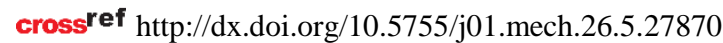

\section{Nomenclature}

$\rho$ is density; $A$ is sectional area; $E$ is Young's modulus; $I_{x}$ is second moment of area; $y$ is the radial displacement of the arch axis; $w$ is the tangential displacement of the arch axis; $\epsilon_{m}$ is strain caused by the change of arch axis length; $\epsilon_{b}$ is strain caused by bending of arch axis; $v_{c}$ is Vault displacement; $L$ is the span of the arch; $f$ is the height of the arch; $m$ is impact mass; $v$ is impact velocity; $t$ is wall thickness.

\section{Introduction}

When an in-plane load is applied suddenly to a shallow circular arch that is fully braced laterally, the load will impart kinetic energy to the arch and will cause the arch to oscillate about an equilibrium position. If this suddenly applied load is sufficiently large, the arch may reach an unstable equilibrium position, which may then induce dynamic buckling of the arch.

The dynamic equation of arches under a sudden central concentrated load is a group of high non-linear differential equations, and the solution is difficulty. There are three main methods used in dynamic researches on arches, including: Solving the dynamic equation to obtain the dynamic response and critical buckling load of arches, numerical method and test method.

The analytic solutions of the arches' dynamic response considering the initial condition and boundary conditions are gotten based on series simplified dynamic differential equations with different hypotheses. Previous investigations on the dynamic buckling of arches are carried out with a rigid-plastic material model assumption and the structural deformation become relatively simple for analysis [1-3]. However, this method is applicable for the dynamic mechanical calculation when the structure undergoes small deformation. Further study [4-5] has been carried out which is suitable for structures with maximum deformation. In order to simplify calculating, the calculation model of arches with 5 hinge joints is set up to analyze the dynamic response of arch under impact load on the vault [6].

It is difficult to establish and solve the dynamic differential equation of arch, so energy method is used in some studies for dynamic property analysis of arches. With energy methods, Donaldson [7] investigated the conditions for maintaining dynamic stability of arches under multiple concentrated loads. In Han Qiang's study [8], snap-through buckling of an elastic shallow arch underground impact is investigated through energy balance equation with Hamiltonian principle and the stability critical state and dynamic response of the structure are given. Based on energy theory, Y. L. Pi [9-12] brought series studies about nonlinear dynamic buckling of arch under different boundary conditions. It is considered that the kinetic energy of arch is zero at the critical dynamic state and the dynamic problem is transformed into a static one and dynamic buckling load is obtained in these studies.

Numerical method is widely used in the analysis of structural dynamic response study [13-14]. Effect of stress wave in structure and local buckling can be obtained with FEM which is difficult to analyze in theoretical calculations. In Ma Xiaotong's study [15], impact test for lattice shells was conducted and dynamic response data of the structure, such as strain, modal and accelerated speed, has been obtained through the corresponding detection means. The experimental results agree well with the numerical results.

Because of the complex mechanical properties of arches and the dynamic differential equations that is hard to get an accurate solution, there is no satisfactory method for dynamic study of arch and different research methods have their own limitations. Considering the advantages of numerical simulation in structural dynamic analysis, researches are carried out with finite element method on dynamic stability critical state of pin-ended arches under a sudden central concentrated load. The dynamic stability analysis of arch is given from energy point of view.

\section{Differential equations of arches' motion and its' solv- ing method}

Pin-ended arch is shown as Fig. 1 and based on the geometrical relationship, the strain energy and kinetic energy of the arch in motion can be obtained. When a central radial load $F$ applied suddenly with infinite duration shown as Fig. 2, the equations of motion can be established by applying the Hamilton's variance principle, which are:

$$
M^{\prime \prime}-\left(N R \tilde{v}^{\prime}\right)^{\prime}-N R+\operatorname{Dirac}(\theta) F R-\rho A R^{3} \ddot{\tilde{v}}=0,
$$




$$
(N R)^{\prime}+\rho A R^{3} \ddot{\tilde{w}}=0,
$$

where:

$$
N=-A E\left(\tilde{w}^{\prime}-\tilde{v}+\frac{\tilde{v}^{\prime 2}}{2}\right) \text { and } M=-\frac{E I_{x} \tilde{v}^{\prime \prime}}{R},
$$

()$^{\prime}=\partial() / \partial \theta$ and $(\dot{)}=\partial() / \partial t, \tilde{v}=v / R$ and $\tilde{w}=w / R$, $v$ and $w$ are he radial displacement and tangential displacement of the arch axis respectively, $R$ is the initial radius of the arc arch. Dirac ( ) is Dirac function.

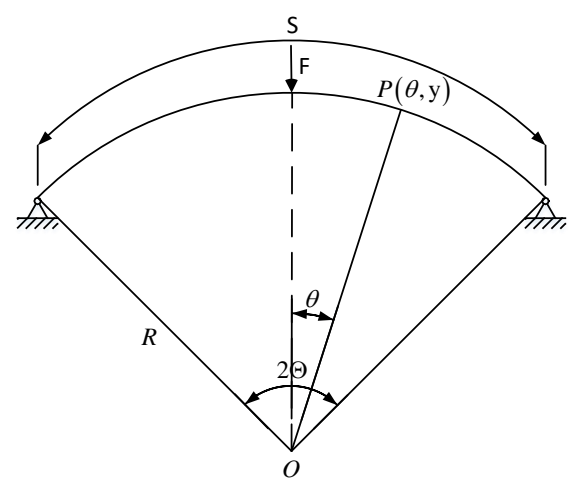

a

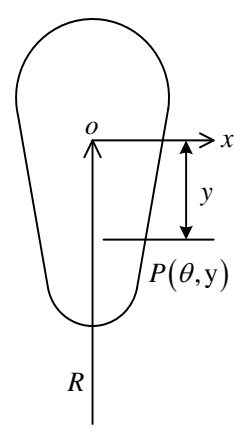

b
Fig. 1 Arch model: $\mathrm{a}$-Arch axis, $\mathrm{b}$-Arch section

Note: $F$ is the central radial load; $S$ is the length of arch axis; $2 \Theta$ is the central angle of the arc

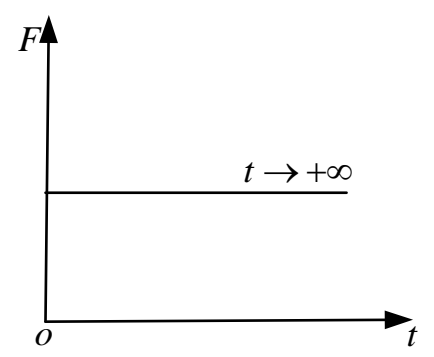

Fig. 2 Sudden load

In Pi's study [9-12], it's proposed that when the arch structure reaches the dynamically stable critical state, the kinetic energy of the structure is very small or even zero, that is, the arch will reach a state of rest at the dynamic Stability state. Hence, the velocity and acceleration in Eqs. (1) and (2) are both equal to zero, and the static equilibrium path can be obtained as shown in Fig. 3 .

Since the strain energy can be obtained based on the geometrical relationship, and the load is known, the total potential energy of the system can be calculated by:

$$
\bar{U}=A_{2} \bar{F}^{2}+B_{2} \bar{F}+C_{2},
$$

where: $\bar{F}=\frac{F R^{2} \Theta}{2 E I_{x}}$, and

$$
A_{2}=\frac{r_{x}^{2}\left[\mu \Theta+4 \mu \Theta \cos ^{2}(\mu \Theta)-5 \sin (\mu \Theta) \cos (\mu \Theta)\right]}{2 \mu^{3} \Theta^{2} R^{2} \cos ^{2}(\mu \Theta)},
$$

where: $\mu^{2}=\frac{N R^{2}}{E I_{x}} \lambda=\frac{R \Theta^{2}}{r_{x}}=\frac{\Theta S}{2 r_{x}}$ and $r_{x}=\sqrt{\frac{I_{x}}{A}}$.

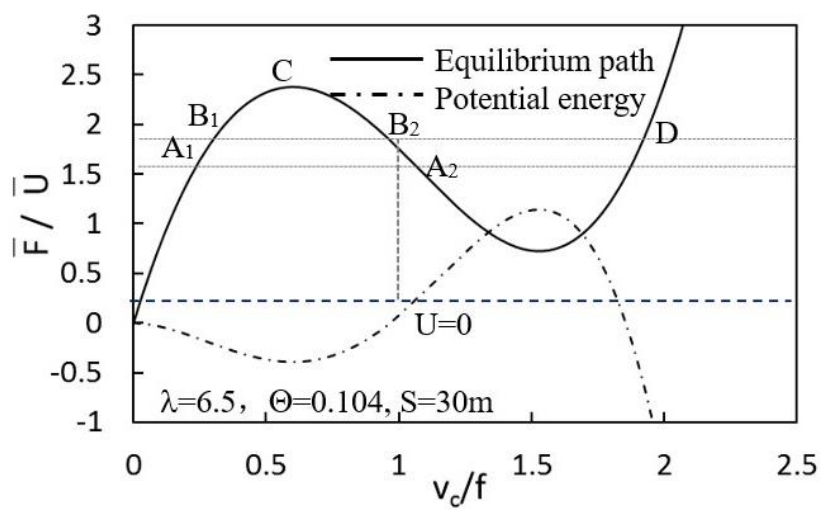

Fig. 3 Load- vault displacement curve and dimensionless total potential energy- vault displacement curve

Dimensionless total potential energy- vault displacement curve calculated by equation (4) is also shown in Fig. 3. The equilibrium path shown in Fig. 3 is divided into stable and unstable equilibrium path by point C. Static instability of the arch will occur on the arch when the arch is in the configuration of point $\mathrm{C}$ under static load. It can be obtained from Fig. 3 that, when the load $\bar{F}$ is relatively small, like $\bar{F}=1.5$, point $A_{1}$ is on the stable equilibrium path and $A_{2}$ is on the unstable equilibrium path. At point $A_{1}$, the configuration of the arch is stable and the total structural potential energy corresponding to this configuration is negative, therefore, the corresponding sudden load can make the arch vibrate at $A_{1}$ 's configuration. However, the total structural potential energy corresponding to the configuration of point $A_{2}$ is positive which is impossible because it violates the law of energy conservation. Therefore, the sudden load of 1.5 will not make the structure lost stability and the structure will vibrate at $A_{1}$ 's configuration.

When $\bar{F}=1.846$, point $B_{1}$ is on the stable equilibrium path and $B_{2}$ is on the unstable equilibrium path. The principle is like $A_{1}$, the arch will vibrate at $B_{1}$ 's configuration but not buckle. The total structural potential energy corresponding to the configuration of point $B_{2}$ is exactly equal 
to 0 , because the law of energy conservation must be satisfied, the kinetic energy at point $B_{2}$ 's configuration should be 0 . Thus, under the sudden load of 1.8406 , the arch will move to the $B_{2}$ 's configuration and exactly stop at that state without instability, and this is the dynamic stability critical state of pin-ended arches under a sudden central concentrated load and $\bar{F}=1.846$ is critical load.

When $\bar{F}$ is greater than 1.8406 , shown as Fig. 3, the total structural potential energy corresponding to the configuration of the stable equilibrium path or the unstable equilibrium path are negative and the kinetic energy corresponding to these configuration are positive. The arch will continue moving until it becomes unstable under a sudden central concentrated load which is greater than the critical load. The point $C$ in Fig. 3 is the static stability critical state and point $B_{2}$ is the dynamic stability critical state of pinended arches, thus, the critical load of dynamic stability under sudden load is less than the critical load of static stability critical state.

The method described above for obtaining the dynamic stability critical state of arch under a central radial sudden load is based on the assumption that the kinetic energy of the arch is almost zero when the configuration of the arch is in the critical state. The critical sudden load can be gotten by solving the equations of motion which is established by applying the Hamilton's variance principle and the total potential energy of the system simultaneously, and the condition of the zero kinetic energy of the arch will be considered in calculation.

\section{Numerical analysis of elastic arch}

The numerical analyses of elastic arches when impact loads applied are carried out in Abaqus/Explicit and the static analysis of that are obtained by Abaqus/Static, RIKS. The arch in the study has enough outside support to ensure that in-plane instability occurs on arch symmetrically. The material property of steel arch is elastic whose Young's modulus is $210 \mathrm{GPa}$ and Poisson ratio is 0.3 .

3.1. Determination on the dynamic stability critical state of elastic arch

The motion of arch under a central radial sudden load can be determined by the vertical displacement varied with time of the node at the arch crown. The arch with solid rectangular section which is in $30 \mathrm{~m}$ arch length is analyzed under different value of sudden load and the time history curves of crown's vertical displacement are shown in Fig.4. The sectional dimension is $0.8 \mathrm{~m} \times 0.906 \mathrm{~m}$.

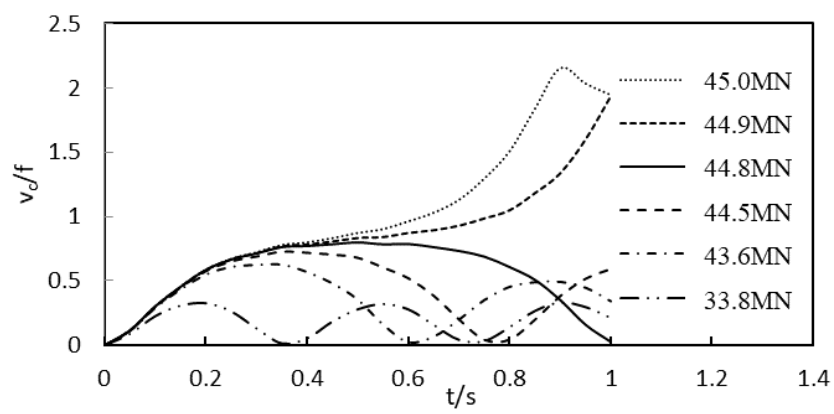

Fig. 4 Time history curves of crown's vertical displacement
From Fig. 4, it could be observed that, the comparatively small dynamic loads won't cause the arch losing steady, but vibrating steadily. With the increasing of the sudden loads' value, the vibration amplitude of the arch crown is increasing and the vibrational frequencies decrease. When the load increases to $4.49 \mathrm{e} 7 \mathrm{~N}$, the time-history curve of the vault displacement deviates from the stable vibration and instability of extreme point type occurs in arch. The motion state of the arch under the sudden load of 4.48e7N shown in Fig. 4 is considered as the dynamic stability critical state and the sudden load of $4.48 \mathrm{e} 7 \mathrm{~N}$ is the critical load. Considering the above analysis, in finite element analysis, the state of arch can be determined by the vertical displacement varied with time and the critical load can be obtained by repeating trial-calculation.

\subsection{Dynamic stability of elastic arch}

Rise-span ratio and slenderness ratio are two major geometric indexes in the structural design of arch which will determine the mechanical properties. The impact of risespan ratio and slenderness ratio on arches' dynamic stability has been studied in this research.

Finite element analyses are carried out for 17 arches with the change range of rise-span ratio from 0.1 to 0.5 . The rise-span ratio is changed with the arch height changing. Geometric parameters except the arch height are constant for finite element examples in this part. Arches are in $30 \mathrm{~m}$ span and the size of arch section is solid and in $0.9 \mathrm{~m} \times 1 \mathrm{~m}$.

The critical load can be obtained by repeating trialcalculation with the determination method described above. The time history curves of crown's vertical displacement calculated by FEM are shown in Fig. 5. Because the trend of the time history curves of crown's vertical displacement are similar when arches are of different dimensions, only curves of arches in 0.1 and 0.5 rise-span ratio are shown in Fig. 5.
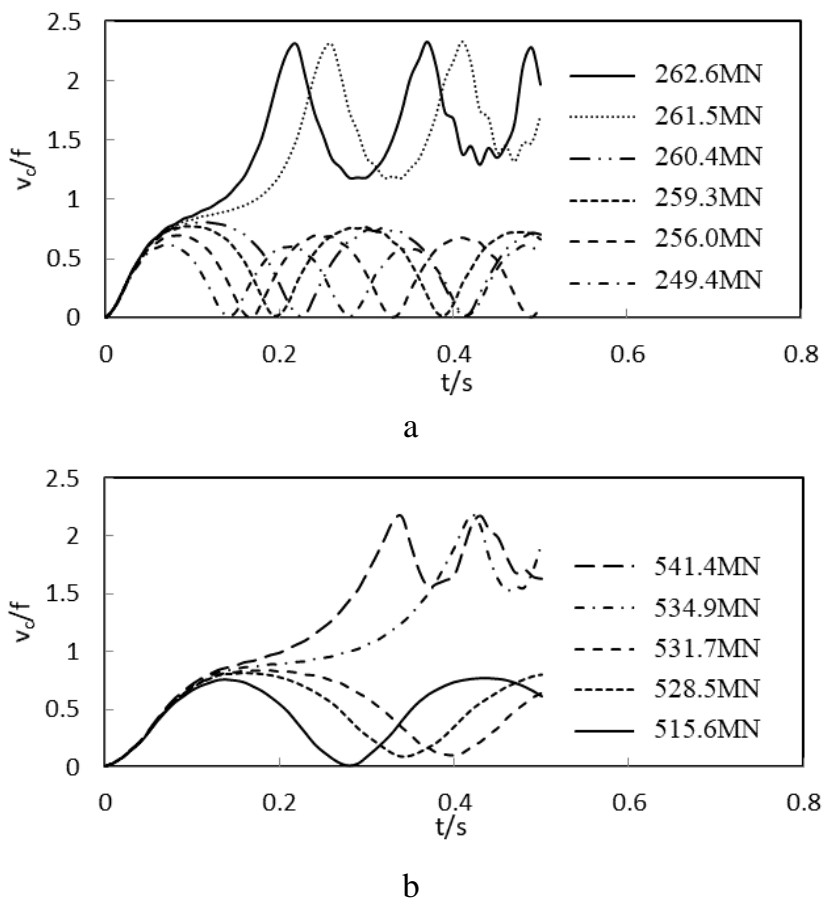

Fig. 5 Time history curves of crown's vertical displacement: $\mathrm{a}-$ Rise-span ratio $=0.1, \mathrm{~b}-$ Rise-span ratio $=0.5$ 
The time history curves of crown's vertical displacement shown in Fig. 5 are similar to that shown in Fig. 4. Thus, the motion of arches analyzed in this section is similar to section 3.2, and the critical loads can be gotten from the vertical motion of crown which are shown in Table 1 . The analytical solutions obtained by the method described in section 2 are also listed in Table 1. The percentages of the difference between finite element solutions and analytical solutions are calculated shown in Table 1 and the percentages of difference are no more than $4.5 \%$. Stress waves transfer and local distortion in arches' motion can be considered in FEM, so the finite element solutions are relatively true and accurate. Therefore, the calculation method of analytic solution introduced in this paper is acceptable.

Table 1

The critical loads of arches with the change range of rise-span ratio $(f / L)$ from 0.1 to 0.5

\begin{tabular}{|c|c|c|c|}
\hline$f / L$ & $\begin{array}{c}\text { Finite element } \\
\text { solutions/MN }\end{array}$ & $\begin{array}{c}\text { Analytical so- } \\
\text { lutions /MN }\end{array}$ & $\begin{array}{c}\text { Percentage of the } \\
\text { difference }(\%)\end{array}$ \\
\hline 0.1 & 260.4 & 262.6 & -0.838 \\
\hline 0.125 & 331.5 & 333.5 & -0.600 \\
\hline 0.15 & 393.7 & 393.3 & 0.102 \\
\hline 0.175 & 447.0 & 443.6 & 0.766 \\
\hline 0.2 & 490.7 & 483.4 & 1.510 \\
\hline 0.225 & 526.5 & 516.0 & 2.035 \\
\hline 0.25 & 554.3 & 544.6 & 1.781 \\
\hline 0.275 & 575.0 & 557.6 & 3.121 \\
\hline 0.3 & 588.5 & 570.7 & 3.119 \\
\hline 0.325 & 595.0 & 573.6 & 3.731 \\
\hline 0.35 & 597.0 & 575.5 & 3.736 \\
\hline 0.375 & 593.1 & 577.7 & 2.666 \\
\hline 0.4 & 583.2 & 562.2 & 3.735 \\
\hline 0.425 & 574.3 & 560.3 & 2.499 \\
\hline 0.45 & 560.3 & 536.8 & 4.378 \\
\hline 0.475 & 547.4 & 534.1 & 2.490 \\
\hline 0.5 & 531.7 & 515.6 & 3.123 \\
\hline
\end{tabular}

The impact of slenderness ratio on arches' dynamic stability is studied through changing the slenderness ratio of the finite element arches' model. The change range of slenderness ratio is from 25 to 100 and the slenderness ratio is changed with the changing of the height of the arch section. Geometric parameters except the arch section's height are constant, the rise-span ratio of arches is 0.15 , the height is $30 \mathrm{~m}$ and the width of arch section is $1 \mathrm{~m}$. Repeating finite element dynamic analyses are performed for 9 arches with different slenderness ratios under central radial sudden loads. The determined method of critical loads and the motion of arches are similar to the above analysis and the critical loads can be gotten from the vertical motion of crown which are shown in Table 2 . The analytical solutions and the percentages of the difference between finite element solutions and analytical solutions are listed in Table 2, too. The percentage of the differences between finite element solutions and analytical solutions in this part are also very small, no more than $1.5 \%$.

It can be observed from Table 1 that, the critical load of dynamic stability increases first and then decreases with the increase in rise-span ratio and the optimum risespan ratio for elastic arches to resist the sudden load is 0.35 .
The critical load of dynamic stability shown in Table 2 decreases with the slenderness ratio increasing.

Table 2

The critical loads of arches with the change range of slenderness ratio $\left(S / i_{x}\right)$ from 25 to 100

\begin{tabular}{|c|c|c|c|}
\hline$S / i_{x}$ & $\begin{array}{c}\text { Finite element } \\
\text { solutions/MN }\end{array}$ & $\begin{array}{c}\text { Analytical so- } \\
\text { lutions } / \mathrm{MN}\end{array}$ & $\begin{array}{c}\text { Percentage of the } \\
\text { difference }(\%)\end{array}$ \\
\hline 25.0 & 3594.0 & 3604.0 & -0.277 \\
\hline 27.5 & 2842.0 & 2856.0 & -0.490 \\
\hline 30.6 & 2177.0 & 2188.0 & -0.503 \\
\hline 34.4 & 1601.0 & 1609.0 & -0.497 \\
\hline 39.3 & 1119.0 & 1121.0 & -0.178 \\
\hline 45.9 & 731.5 & 729.7 & 0.247 \\
\hline 55.0 & 437.4 & 434.1 & 0.760 \\
\hline 68.8 & 230.2 & 227.3 & 1.276 \\
\hline 91.7 & 99.0 & 97.5 & 1.497 \\
\hline
\end{tabular}

3.3. Energy characteristics of elastic arch when instability occurs

The assumption has been proposed in theoretical analysis as described above, that when the arch structure reaches the dynamically stable critical state, the kinetic energy of the structure is very small or even zero. The kinetic energy of arches when its reach the dynamically stable critical state calculated by FEM with the arches' model in above section are listed in Table 3. The strain energy of arches at the dynamically stable critical state are also listed in Table 3 and it could be observed that the kinetic energy is much smaller than strain energy, the maximum ratio is no more than $0.8 \%$. Therefore, the assumption in the analytical solution that the kinetic energy is zero in the dynamically stable critical state is reasonable.

Table 3

Kinetic energy and strain energy of arches at dynamically stable critical state

\begin{tabular}{|c|c|c|c|}
\hline$f / L$ & $K E N / \mathrm{J}$ & SEN/J & KEN / SEN $(\%)$ \\
\hline 0.1 & 3620790 & 625768000 & 0.579 \\
\hline 0.125 & 3073200 & 993942000 & 0.309 \\
\hline 0.15 & 3643120 & 1479430000 & 0.246 \\
\hline 0.175 & 2938290 & 1968200000 & 0.149 \\
\hline 0.2 & 2375590 & 2421000000 & 0.098 \\
\hline 0.225 & 8754480 & 3022020000 & 0.290 \\
\hline 0.25 & 11331900 & 3567690000 & 0.318 \\
\hline 0.275 & 4172480 & 4249180000 & 0.098 \\
\hline 0.3 & 13280600 & 4732570000 & 0.281 \\
\hline 0.325 & 13771800 & 4980670000 & 0.277 \\
\hline 0.35 & 20233700 & 5399000000 & 0.375 \\
\hline 0.375 & 25959600 & 5622430000 & 0.462 \\
\hline 0.4 & 16248700 & 5698160000 & 0.285 \\
\hline 0.425 & 25719400 & 6024690000 & 0.427 \\
\hline 0.45 & 34139800 & 6103770000 & 0.559 \\
\hline 0.475 & 44235100 & 6302740000 & 0.702 \\
\hline 0.5 & 17040000 & 6632490000 & 0.257 \\
\hline
\end{tabular}

Note: $K E N$ represent kinetic energy at dynamically stable critical state; $S E N$ represent strain energy at dynamically stable critical state. 


\section{Numerical analysis of elastic-plastic arches}

The dynamic response for elastic-plastic arches is more complicated than elastic arches. Numerical simulation method is also used in dynamic stability study of elasticplastic arches in this section. The material property of arches in this section is perfect elastic-plastic steel whose Young's modulus is $210 \mathrm{GPa}$, yield strength is $235 \mathrm{MPa}$ and Poisson ratio is 0.3 .

\subsection{Determination on the dynamic stability critical state of} elastic-plastic arch

It is found by repeating trial-calculation on elasticplastic arch that the dynamic stability critical state under a central radial load applied suddenly could also be determined by the vertical displacement of crown varied with time as in the elastic arches' analysis. Taking the arch with a rise-span ratio of 0.1 as an example, the time-history curves of vertical displacement of crown under different sudden loads is shown in Fig. 6.

It can be observed from Fig. 6 that the time history curves of crown's vertical displacement under different loads almost coincide with each other at least initially, and when the sudden load is relatively small, such as $2.9 \mathrm{e} 7 \mathrm{~N}$, the arch vibrates with a small amplitude at a certain distance from the initial position. With the increase of the sudden load, the maximum displacement of the crown of the arch increases and the vibration amplitude decreases. When the sudden load increases to $2.949 \mathrm{e} 7 \mathrm{~N}$, the time history curve of crown's vertical displacement is approximate a horizontal line shown as Fig. 6. A sudden load greater than $2.949 \mathrm{e} 7 \mathrm{~N}$ will cause the arch lose stability, which is shown as the arch crown move out of the limit position shown in Fig. 6. Thus, the sudden load $2.949 \mathrm{e} 7 \mathrm{~N}$ is the critical load of the arch through the repeating trial-calculation.

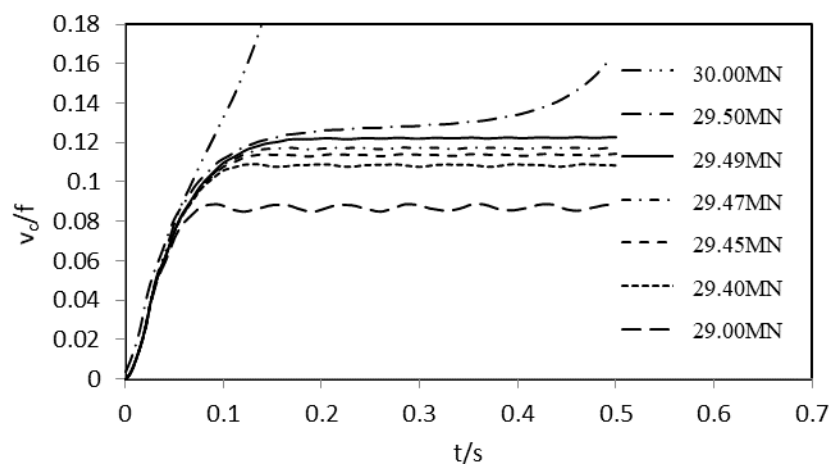

Fig. 6 Time history curves of crown's vertical displacement

The expanding law of plastic region and the deformation characteristics of the arches under a central radial load applied suddenly are shown as Fig. 7. From Figs. 7A to $7 \mathrm{D}$, it shows that the state of arch is from the beginning of the sudden load applied to structural instability occurred. The arch worked in elastic behavior before the state shown as Fig. 7A reached and the crown's vertical displacement is small. The arch's crown yields firstly at the state shown as Fig. 7A and with the structural configuration changes shown as Fig. 7B. The force state of the arch is changing from state A to state B which cause the internal force redistribution and the plastic region changing. When the structure moves to the state shown as Fig. 7B, the stiffness of the structure decreased significantly and the plastic region expands rapidly to the state shown as Fig. $7 \mathrm{C}$, and then, the plastic region expansion basically stops. But the crown's vertical displacement is still increasing until the plastic hinges is formed at $1 / 4$ and 3/4 span shown as Fig. 7D. The structure moves around the plastic hinge after the state shown as Fig. 7D, and crown's vertical displacement increase rapidly accompanied by structural instability.

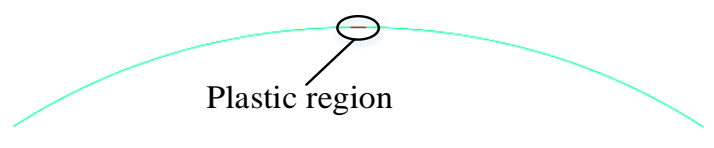

A

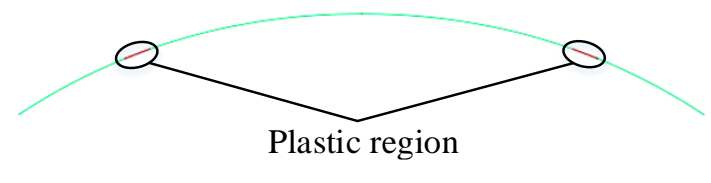

B
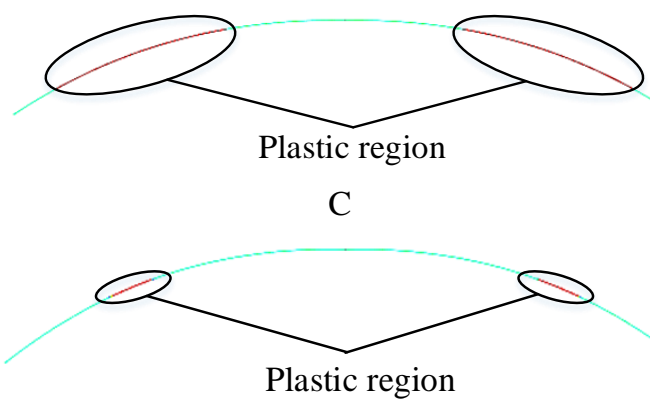

$\mathrm{D}$

Fig. 7 The plastic region on arch

The elastic strain energy variation regular of elastic-plastic arch under sudden load is analyzed around the dynamic instability process considering plastic region development characteristics as discussed above. The relationship of the finite element analysis results of elastic strain energy with crown's vertical displacement is shown in Fig. 8.

The curve shown in Fig. 8, a can be divided into three parts which are the rising part OM, flat part $\mathrm{MN}$ and the descent part NP. Point M is the peak point on the strain energy curve, and the plastic hinge of the arch forms when it reaches point $M$ which is shown as Fig. 7D. The elastic strain energy of the arch won't increase after point $\mathrm{M}$ and the crown's vertical displacement is increasing until the structure moves to the state of point $\mathrm{N}$. After point $\mathrm{N}$, the curve in Fig. 8, a shows that the elastic strain energy release rapidly and the bearing capacity of the arch lose completely. The appearance of plastic hinges on the arch shown as Fig. 7D means that the structure lose stability although the structure still has a higher bearing capacity.

When different sudden central radial loads are applied, the relationship of elastic strain energy with crown's vertical displacement are similar and the curves of elastic strain energy changing with the displacement of the vault all have the rising part OM, flat part MN shown as Fig. 8, a, the difference of them is whether there is a descent part like NP. When the sudden load is relatively small which won't lead to the instability of the arch, the arch vibrates at a certain distance from the initial position and in that state, the elastic strain energy of the arch is maximum and stable whose value is less than the peak point' value on the strain energy curve of the lost stability arch shown as Fig. 8, a. When the 
arch moves to the dynamic stability critical state under critical load, because the arch is almost motionless, the curve of elastic strain energy with the crown's displacement still don't have the descent part, and the elastic strain energy of the arch in that critical state is the maximal no matter how much the sudden load increases.

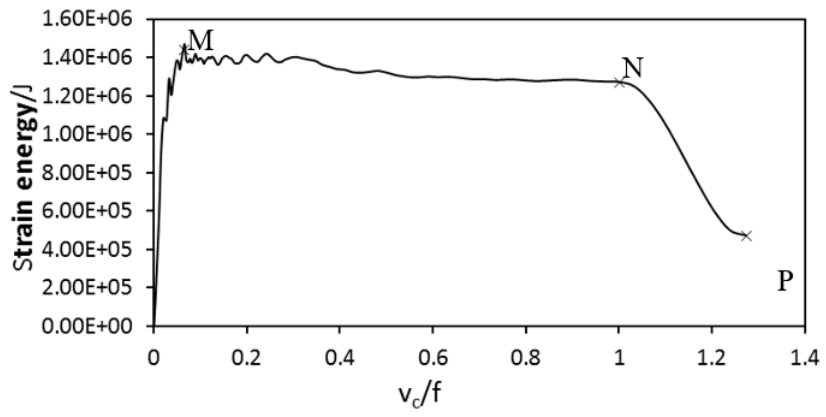

a

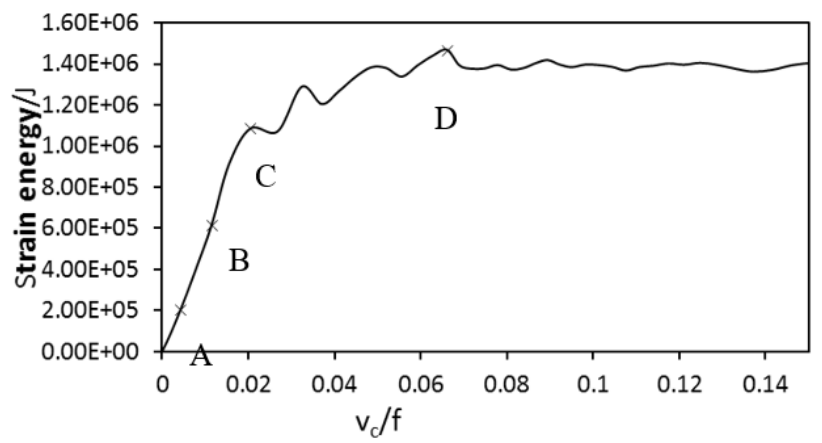

b

Fig. 8 Elastic strain energy of elastic-plastic arch under a sudden load applied: a - overall curve, b - partial enlarged curve

The rising part OM shown in Fig. 8, a is enlarged in Fig. 8, b and the state of point A, B, C, and D are corresponding to the state shown in Fig. 9.

\subsection{Dynamic stability of elastic-plastic arch}

The dynamic stability of elastic-plastic arch is analyzed with finite element method. The impact of rise-span ratio and slenderness ratio on elastic-plastic arches' dynamic stability has been studied in this section. 9 elasticplastic arches with the change range of rise-span ratio from 0.1 to 0.5 have been analyzed with FEM. The rise-span ratio is changed with the arch height changing, arches are in constant span of $30 \mathrm{~m}$ and the size of arch section is $0.8 \mathrm{~m} \times 1 \mathrm{~m}$ constantly.

When studying the effects of slenderness ratio on the dynamic stability of elastic-plastic arch, the finite element models analyzed are in constant rise-span ratio of 0.15 , constant span of $30 \mathrm{~m}$ and the width of arch section is $1 \mathrm{~m}$ without changing. The change range of slenderness ratio is from 25 to 100 and the slenderness ratio is changed with the changing of the height of the arch section.

The critical load can be obtained by repeating trialcalculation and the results are shown in Fig. 9. It can be observed from Fig. 9, a that the critical load of dynamic stability of elastic-plastic arch increases first and then decreases with the increase in rise-span ratio and the optimum risespan ratio for elastic-plastic arches to resist the sudden load is 0.2 . The critical load of dynamic stability shown in Fig. 9 , b decreases with the slenderness ratio increasing.
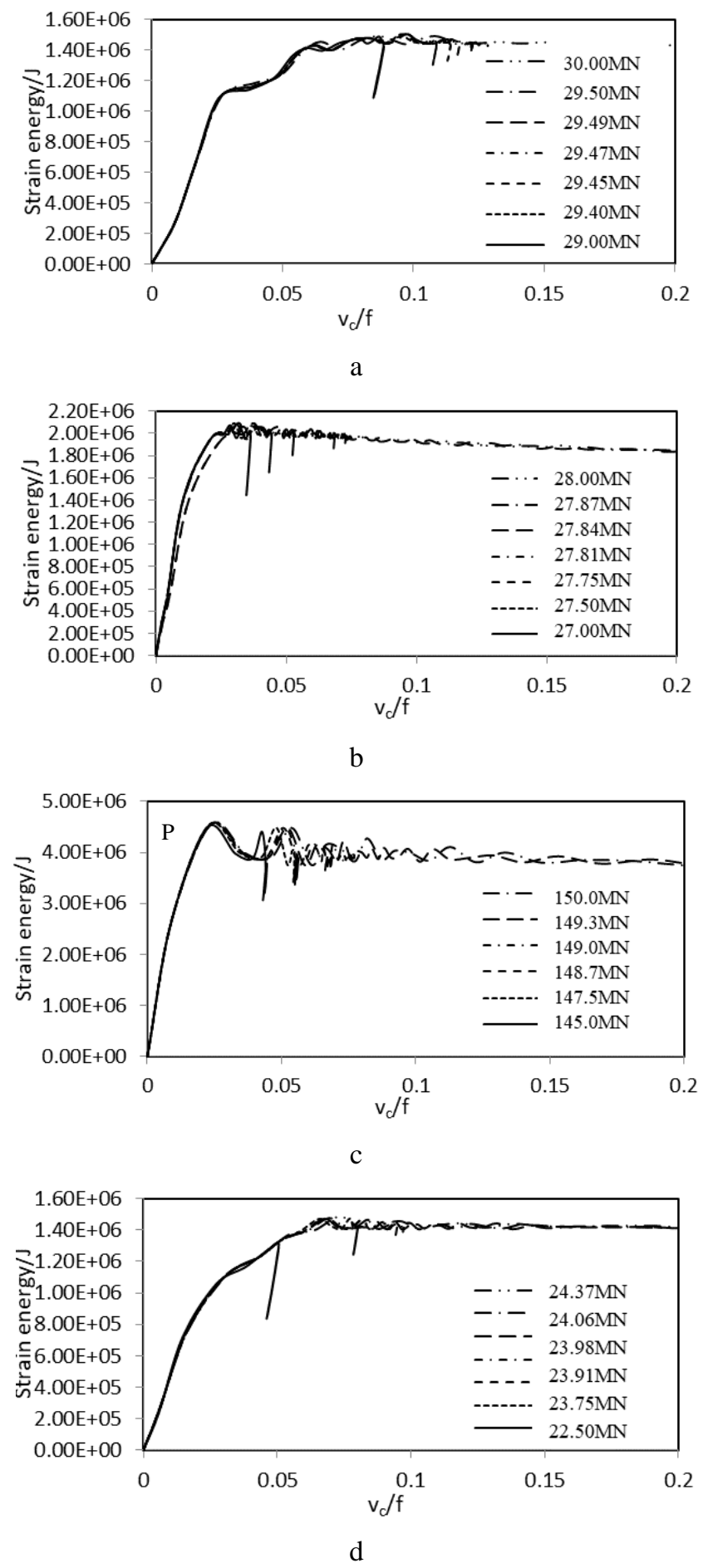

Fig. 9 Elastic strain energy of elastic-plastic arch under a sudden load applied: a - rise-span ratio $=0.1, \mathrm{~b}-$ risespan ratio $=0.4, \mathrm{c}-$ rise-span ratio $=25.0, \mathrm{~d}$ - rise-span ratio $=68.8$

The relationship between elastic strain energy and crown's vertical displacement under the critical load applied are shown as a black solid line in Fig. 10. The curves shown in Fig. 10 are all for the arches which have lost stability already or reach the dynamic stability critical state. It can be observed from Fig. 10 that for the same model, the curves gotten under different loads are basically the same in trend and value, and maximal elastic strain energies are almost the 
same. So reasonable guess is made which is that the maximal elastic strain energies of an elastic-plastic arch under a central radial load is certain in both static load and sudden load.

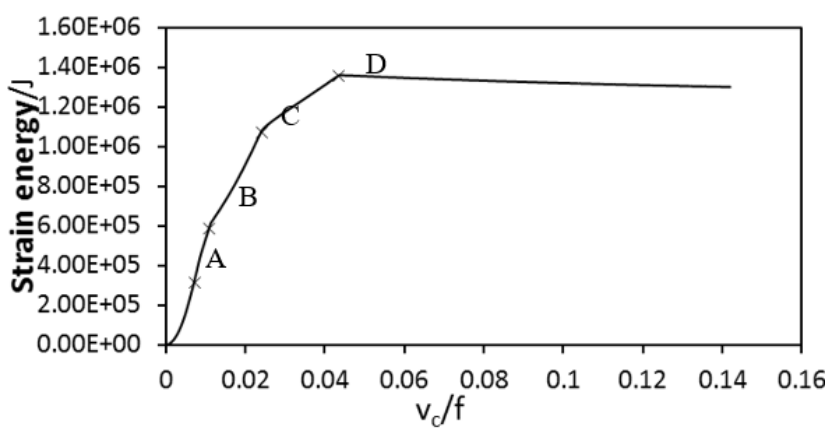

Fig. 10 Elastic strain energy of elastic-plastic arch under static load applied

The relationship between elastic strain energy and crown's vertical displacement under a central radial static load applied are shown in Fig. 10. The curve shown in Fig. 10 is similar with the curve shown in Fig. 8 in trend and the state A, B, C, D shown in Fig. 8 can also be found during the static load process. The elastic strain energy of elasticplastic arches at the dynamic stability critical state are listed in Table 4 and compared with the maximal elastic strain energy in static calculation. It could be observed from the percentage of the difference between elastic strain energy of elastic-plastic arches at the dynamic stability critical state and the maximal elastic strain energy in static calculation shown in Table 4 that the difference is no more than $3.5 \%$. Thus, the maximal elastic strain energy in static calculation can be used in determining the state of the elastic-plastic arch under sudden loads applied and this method is more accurate and easy to realize.

Table 4

Elastic strain energy of elastic-plastic arch at stability critical state

\begin{tabular}{|c|c|c|c|c|c|c|}
\hline$S / \mathrm{m}$ & $f / \mathrm{m}$ & $A / \mathrm{m}$ & $F_{\text {critical }} / \mathrm{kN}$ & $D S E N / \mathrm{J}$ & $S S E N / \mathrm{J}$ & Error $(\%)$ \\
\hline 30 & 3 & $0.9 \times 1$ & 29490 & 1439490 & 1443020 & -0.245 \\
\hline 30 & 4.5 & $0.9 \times 1$ & 33010 & 1551390 & 1530300 & 1.378 \\
\hline 30 & 6 & $0.9 \times 1$ & 34130 & 1704860 & 1669950 & 2.090 \\
\hline 30 & 7.5 & $0.9 \times 1$ & 33320 & 1786670 & 1825820 & -2.144 \\
\hline 30 & 9 & $0.9 \times 1$ & 31660 & 1804370 & 1867980 & -3.405 \\
\hline 30 & 10.5 & $0.9 \times 1$ & 29810 & 1898580 & 1928900 & -1.572 \\
\hline 30 & 12 & $0.9 \times 1$ & 27840 & 1969340 & 2014830 & -2.258 \\
\hline 30 & 13.5 & $0.9 \times 1$ & 25930 & 2071040 & 2118210 & -2.227 \\
\hline 30 & 15 & $0.9 \times 1$ & 24100 & 2175560 & 2234860 & -2.653 \\
\hline 30 & 4.5 & $2.2 \times 1$ & 149000 & 3955780 & 4046070 & -2.232 \\
\hline 30 & 4.5 & $2.0 \times 1$ & 127100 & 3497230 & 3570880 & -2.063 \\
\hline 30 & 4.5 & $1.8 \times 1$ & 106200 & 3077820 & 3130560 & -1.685 \\
\hline 30 & 4.5 & $1.6 \times 1$ & 86570 & 2693020 & 2725300 & -1.184 \\
\hline 30 & 4.5 & $1.4 \times 1$ & 68270 & 2350330 & 2354060 & -0.158 \\
\hline 30 & 4.5 & $1.2 \times 1$ & 51580 & 2032330 & 2014430 & 0.889 \\
\hline 30 & 4.5 & $1.0 \times 1$ & 36680 & 1725860 & 1700230 & 1.507 \\
\hline 30 & 4.5 & $0.8 \times 1$ & 23910 & 1418540 & 1402290 & 1.159 \\
\hline 30 & 4.5 & $0.6 \times 1$ & 13260 & 1064010 & 1085560 & -1.985 \\
\hline
\end{tabular}

Note: DSEN represent dynamically stability critical elastic strain energy; SSEN represent maximal elastic strain energy in static calculation; Error $=(D S E N-S S E N) / S S E N$
The relationship between elastic strain energy and crown's vertical displacement under the critical load applied are shown as a black solid line in Fig. 10. The curves shown in Fig. 10 are all for the arches which have lost stability already or reach the dynamic stability critical state. It can be observed from Fig. 10 that for the same model, the curves gotten under different loads are basically the same in trend and value, and maximal elastic strain energies are almost the same. So reasonable guess is made which is that the maximal elastic strain energies of an elastic-plastic arch under a central radial load is certain in both static load and sudden load.

\section{Impact test on arch}

It has been found from analysis above that the instability of elastic-plastic arch can be judged by the forming of plastic hinges at about $1 / 4$ on both sides of the arch and this conclusion is proved by the impact test on square steel tube arches by rigid bodies in this section.

\subsection{Material test of square steel tube}

According to the provisions in the specification [16], the pipe of a certain length, which is $600 \mathrm{~mm}$, is taken as the material test specimen, and the clamping places at the pipe ends is filled with metal blocks shown as Fig. 11.

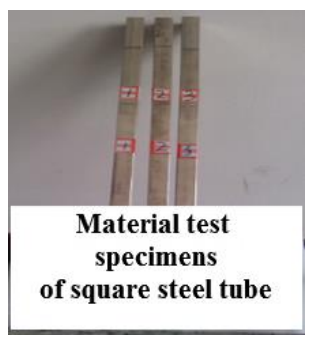

Fig. 11 Test specimen

The measured elastic modulus of the square steel tube is $2.10 \mathrm{e} 11 \mathrm{~Pa}$, the yield stress is $270.4 \mathrm{MPa}$, the ultimate stress is $311.1 \mathrm{MPa}$, and the failure strain is 0.056 . It can be obtained that the true yield stress of the square steel tube is $270.7 \mathrm{MPa}$, the true limit stress is $311.4 \mathrm{MPa}$, and the true failure strain is 0.055 .

\subsection{Impact test}

The impact test of steel arch specimen was carried out by using drop-testing machine and device for fixing the supports of arches shown as Fig. 12.

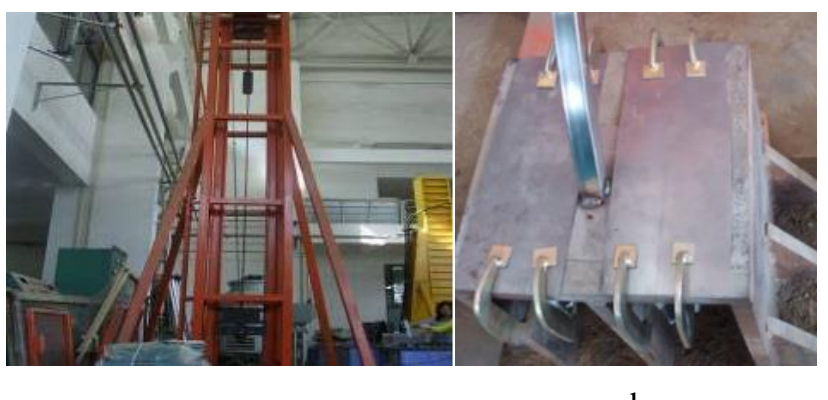

a

b

Fig. $12 \mathrm{a}$-drop-testing machine, $\mathrm{b}$-device for fixing the supports 
There are 9 specimens in the impact test, and the parameters are shown in Table 5. The steel arch specimen is shown in the Fig. 13. The impact velocity is changed by changing the falling height of the drop hammer. During the test, the end plate of the support was fixed on the steel support with a u-shaped clamp to ensure the boundary condition of the fixed support as far as possible. The increased height of the support also reserved deformation space for the overall buckling of the specimen shown as Fig. 12, b.

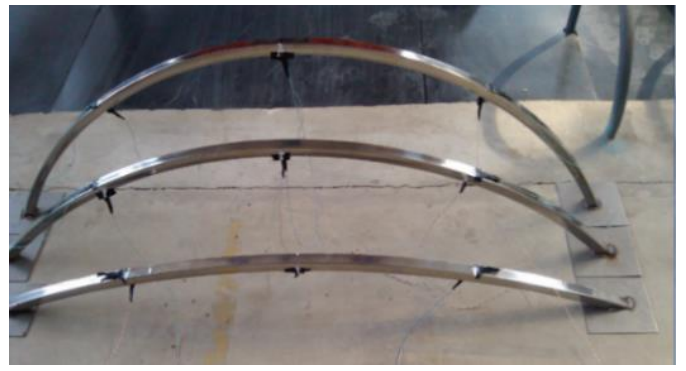

Fig. 13 Steel arch specimen

Parameters of specimens

Table 5

\begin{tabular}{|c|c|c|c|c|c|c|}
\hline NO. & $v, \mathrm{~m} / \mathrm{s}$ & $m, \mathrm{~kg}$ & $L, \mathrm{~m}$ & $f / L$ & $A, \mathrm{~mm} \times \mathrm{mm}$ & $t, \mathrm{~mm}$ \\
\hline $1-1$ & 4 & 35 & 2 & 0.1 & $22 \times 22$ & 1 \\
\hline $1-2$ & 4 & 35 & 2 & 0.2 & $22 \times 22$ & 1 \\
\hline $1-3$ & 4 & 35 & 2 & 0.3 & $22 \times 22$ & 1 \\
\hline $2-1$ & 3 & 35 & 1.5 & 0.1 & $22 \times 22$ & 1 \\
\hline $2-2$ & 3 & 35 & 1.5 & 0.2 & $22 \times 22$ & 1 \\
\hline $2-3$ & 3 & 35 & 1.5 & 0.3 & $22 \times 22$ & 1 \\
\hline $3-1$ & 3 & 35 & 1 & 0.1 & $22 \times 22$ & 1 \\
\hline $3-2$ & 3 & 35 & 1 & 0.2 & $22 \times 22$ & 1 \\
\hline $3-3$ & 3 & 35 & 1 & 0.3 & $22 \times 22$ & 1 \\
\hline
\end{tabular}

\subsection{Test results}

Through monitoring the strain at the middle arch span and $1 / 4$ on both sides of the arch during the impact process, it is found that all the monitoring points of all specimens enter the yield after the impact. In-plane instability occurred in all specimens and its deflection shapes after buckling shown as Fig. 14.
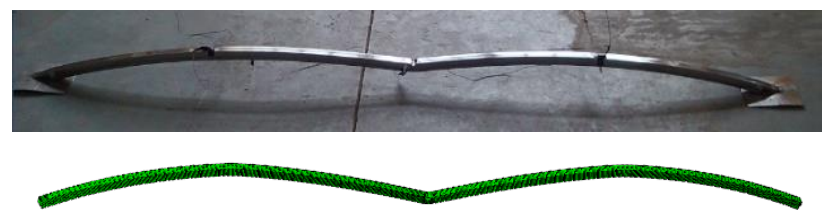

a

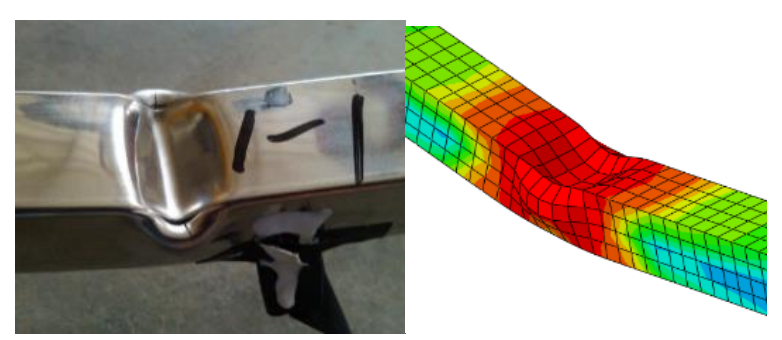

b

Fig. 14 Comparison of failure modes between experiment and numerical simulation of specimen 1-1: a - Comparison of deflection shapes between experiment and numerical simulation, $\mathrm{b}$ - Comparison of local failure modes between experiment and numerical simulation
By observing the failure modes of 9 specimens combined with the yield situation of the measuring point, the relevant laws can be summarized as follows: (1) the yield locations on the specimens are usually located at the impact point and about 1/4 of the span from the arch foots; (2) local dent occurred at the impact point; (3) In-plane instability occurred in all specimens. The instability mode of the arch under impact shown in experiment is the same as that in numerical simulation, and the failure mode between them are similarity. Therefore, the numerical simulation can well predict the dynamic stability of the arch.

\section{Conclusion}

The method for determining the critical state of dynamic stability of arch under a sudden central concentrated load are proposed in this study based on numerical simulation analysis which considered the material and geometric nonlinear property. The specific work and conclusions are as the follows:

1. According to the FEM analyses of elastic arches when central radial loads applied suddenly, the state of arches can be determined by the crown's vertical movement and the critical load can be obtained by repeating trial-calculation. Through the simulation results of elastic arches with changing geometric parameters, it is found that the optimum rise-span ratio for elastic arches to resist the sudden load is 0.35 and the critical load of dynamic stability of elastic arch decreases with the slenderness ratio increasing.

2. The existing theoretical analysis method for obtaining the dynamic critical load of elastic arch under central radial sudden load is established by applying the Hamilton's variance principle, and the assumption has been proposed in theoretical analysis that when the arch structure reaches the dynamically stable critical state, the kinetic energy of the structure is very small or even zero. The assumption about the kinetic energy has been proved to be reasonable with the finite element calculation results in this study, and the dynamic critical load calculated with the theoretical analysis method is accuracy enough compared with the finite element calculation results. The percentage of the differences between finite element solutions of critical load and analytical solutions are no more than $4.5 \%$.

3 . It is found by repeating trial-calculation on elastic-plastic arch that the dynamic stability critical state under a central radial load applied suddenly could also be determined by the vertical displacement of crown varied with time as in the elastic arches' analysis. The optimum risespan ratio for elastic-plastic arches to resist the sudden load is 0.2 and the critical load of dynamic stability decreases with the slenderness ratio increasing.

4. The expanding law of plastic region and the deformation characteristics of elastic-plastic arch are analyzed. The elastic strain energy reach the maximum value at the appearance of plastic hinges at about $1 / 4$ of the span from the arch foots during the motion process. The finite element results show that maximal elastic strain energy is certain for the arch in a certain geometry under both a sudden load and static load. The maximal elastic strain energy in static calculation can be used to determine the state of the elastic-plastic arch under sudden loads applied and this method is more accurate which errors won't exceed 3.5\%. 


\section{Acknowledgments}

This work was financially supported by National Science Foundation of China (51279206).

\section{References}

1. Parkes, E. W. 1955 . The permanent deformation of a cantilever struck transversely at its tip, Proceedings of the Royal Society of London, Series A, Mathematical and Physical Sciences 228(1175): 462-476. http://dx.doi.org/10.2307/99638.

2. Prager, W. 1959. An introduction to plasticity. Addison-Wesley Pub. Co. http://dx.doi.org/10.1063/1.3056868.

3. Hodge, P G. 1959. Plastic Analysis of Structure. New York: McGraw-Hill. http://dx.doi.org/10.1115/1.3644008.

4. Jones, N. 1989. Recent studies on the dynamic plastic behavior of structures, Applied Mechanics Reviews 238(42): 95-115.

http://dx.doi.org/ 10.1115/1.3152425.

5. Palomby, C.; Stronge, W. J. 1988. Evolutionary modes for large deflections of dynamically loaded rigid-plastic structures, Mechanics of Structures and Machines 16(1): 53-80. http://dx.doi.org/ 10.1080/08905458808960253.

6. Wei, D. M. 2004. Nonlinear Theory of Arch and its Application. Beijing: Science Press.

7. Donaldson, M. T., Plaut, R. H. 1983. Dynamic stability boundaries for a sinusoidal shallow arch under pulse loads, AIAA Journal 21(3): 469-471. http://dx.doi.org/ 10.2514/3.8097.

8. Han, Q.; Huang, H. W.; Fan, X. J. 2010. Nonlinear dynamic buckling of shallow elastic arch. Huanan Ligong Daxue Xuebao, Journal of South China University of Technology (Natural Science) 38(3): 1-7. http://dx.doi.org/10.3969/j.issn.1000565X.2010.03.001.

9. Pi, Y. L.; Bradford, M. A. 2008. Dynamic buckling of shallow pin-ended arches under a sudden central concentrated load, Journal of Sound and Vibration 317(35): 898-917. http://dx.doi.org/10.1016/j.jsv.2008.03.037.

10. Pi, Y. L.; Bradford, M. A. 2010. Effects of prebuckling analyses on determining buckling loads of pin-ended circular arches, Mechanics Research Communications 37(6): 545-553.

http://dx.doi.org/ 10.1016/j.mechrescom.2010.07.016.

11. Pi, Y. L.; Bradford, M. A.; Tong, G. S. 2010. Elastic lateral-torsional buckling of circular arches subjected to a central concentrated load, International Journal of Mechanical Sciences 52(6): 847-862. http://dx.doi.org/ 10.1016/j.ijmecsci.2010.02.003.

12. Pi, Y. L.; Bradford, M. A.; Tin-Loi, F; Gilbert, R. I. 2007. Geometric and material nonlinear analyses of elastically restrained arches, Engineering Structures 29(3): 283-295

http://dx.doi.org/10.1016/j.engstruct.2006.01.016.
13. Zhang, G.; He, Y.; Ju, J. S.; Jiang, X. G.; Liu, C.; Hou, Z. 2011. Testing and numerical analysis of elastoplastic steel column impacted by rigid body, Journal of Computational \& Theoretical Nanoscience 4(8): 29512956. http://dx.doi.org/ 10.1166/asl.2011.1694.

14. Ju, J. S.; Ding, M.; Shi, X. D.; Cen, S.; Jiang, X. G.; Chen, X. H. 2011. Effect of beam height on elastic impact load subjected to transverse impact of bar, Key Engineering Materials 462-463: 259-264. http://dx.doi.org/10.4028/www.scientific.net/KEM.462-463.259.

15. Ma, X.; Wang, X.; Bao, C.; Lu, H.; Yang, W. 2019. Dynamic response analysis and model test research on k6 single-layer spherical reticulated shells subjected to impact load, International Journal of Steel Structures. http://dx.doi.org/ 10.1007/s13296-019-00221-7.

16. Metallic Materials Tensile Testing at Ambient Temperature. GB-T2282002. 20 p.

K. Qin, J. Ju, J. Li, M. Liu

\section{DYNAMIC STABILITY CRITICAL STATE OF PIN- ENDED ARCHES UNDER SUDDEN CENTRAL CONCENTRATED LOAD}

S u m m a r y

The dynamic in-plane instability process of extreme point type for pin-ended arches when a central radial load applied suddenly with infinite duration is analyzed with finite element method in this study. The state of arch can be determined by the crown's vertical displacement varied with time and the critical load can be obtained by repeating trialcalculation. When the arch structure reaches the dynamically stable critical state, the kinetic energy of the structure is very small or even zero. The dynamic critical load of elastic arch calculated with the theoretical analysis method which is based on energy principle is proved accuracy enough by comparing with the finite element calculation results and the percentage of the differences between them are no more than $4.5 \%$. The maximal elastic strain energy is certain for the elastic-plastic arch in certain geometry under both a sudden load and static load. The maximal elastic strain energy in static calculation can be used in determining the state of the elastic-plastic arch under dynamic sudden loads applied and this method is more accurate which errors won't exceed $3.5 \%$. The accuracy of dynamic critical load calculation method for elastic arch is verified by numerical calculation in this study, and based on the characteristic of elastic strain energy in critical state, a method for determining the stability of elastic-plastic arch is presented.

Keywords: arch; dynamic buckling; energy method; material nonlinearity.

Received September 30, 2019 Accepted October 14, 2020 